\title{
Revisiting WFQ: Minimum Packet Lengths Tighten Delay and Fairness Bounds
}

\author{
Anirudha Sahoo and D. Manjunath
}

\begin{abstract}
In this paper we consider the bounds on the sample path discrepancy between the 'idealized' generalized processor sharing (GPS) and the 'practical' weighted fair queueing (WFQ) scheduling disciplines. We show that when both the minimum packet lengths and the weights are non zero, the discrepancy bounds can possibly be tighter than that in [1] and [2]. This new upper bound on the delay discrepancy is then used to provide an upper bound on the discrepancy in the instantaneous throughput, which can also be significantly tighter than those in [1] and [2].
\end{abstract}

Index Terms-Scheduling, Weighted Fair Queueing (WFQ), Generalized Processor Sharing (GPS), fairness bound.

\section{INTRODUCTION}

G ENERALIZED processor sharing (GPS) generalizes the ideal of processor sharing (PS) and is a max-min fair way of sharing link capacity. Weighted fair queueing (WFQ) was first proposed as an emulation of the PS in [3] and its fairness issues were first analyzed in [1]. By assuming a maximum packet length, they obtained a bound on the discrepancy, the difference in the instantaneous throughput of a flow between the ideal GPS and the WFQ systems. It can be shown that this is the best discrepancy possible by a non preemptive scheduler. Since the instantaneous throughput difference between GPS and WFQ is bounded, it follows that the instantaneous queue lengths differences are also bounded. They also bound the difference in the delays in the WFQ and the GPS systems. In a widely cited paper, Parekh and Gallager [2] refer to WFQ as packetized-GPS or PGPS, and provide fairly simplified proofs for the delay and the fairness bounds between a GPS and the PGPS or WFQ systems. This is now considered the standard proof, e.g., see [4]. Since the above seminal papers, many variations of WFQ have been introduced, e.g., [5]-[9] that address implementation complexity and provide other useful properties.

To the best of our knowledge, all analyses of PGPS, WFQ and related schedulers consider only the maximum packet length in describing the discrepancy and none of them consider the case of a link also prescribing a minimum packet length. This is important because most networks do prescribe such a minimum corresponding to, for example, the header and the trailer lengths. In this paper, we show that for two important performance measures-delay and instantaneous throughput,

Manuscript received October 13, 2006. The associate editor coordinating the review of this letter and approving it for publication was Dr. Alex Sprintson.

A. Sahoo is with the Kanwal Rekhi School of Information Technology, Indian Institute of Technology Bombay, Powai, Mumbai - 400076, India (email: sahoo@it.iitb.ac.in).

D. Manjunath is with the Department of Electrical Engineering, Indian Institute of Technology Bombay, Powai, Mumbai - 400076, India (e-mail: dmanju@ee.iitb.ac.in).

Digital Object Identifier 10.1109/LCOMM.2007.061677. the discrepancy between the GPS and WFQ (or PGPS) is much tighter when the link also prescribes a minimum packet length. Recall that the latter is also a relative fairness measure.

In the next section, we first derive an upper bound on the delay discrepancy when the minimum packet length is non zero. We will see that the correction to the well known bound of [1] and [2] can be significant. The new upper bound on the delay discrepancy is then used to provide an upper bound on the discrepancy in the instantaneous throughput, which is also significantly tighter than those in [1] and [2]. We conclude in Section III with examples and a brief discussion.

\section{DisCREPANCY ANALYSIS With Minimum PaCKet LengthS}

Recall that in the WFQ system, scheduling instants occur at the departure times (end of transmission). The new packet that is scheduled for transmission is the one that would have departed earliest in the corresponding GPS system from among those that are present in the system at the scheduling instant.

We consider a link of capacity $C$ serving $N$ flows indexed $i=1, \ldots, N$. The packets from each flow are served according to FIFO. Let $L_{\min }$ and $L_{\max }$ be the minimum and maximum packet lengths that the link supports. Let $\phi_{i}$ be the weight of flow $i$. Recall that GPS and WFQ guarantee a minimum rate of $\frac{\phi_{i}}{\sum_{i} \phi_{i}}$ to flow $i$. Let

$$
\phi_{\min }:=\min _{i} \phi_{i}, \quad \phi_{\max }:=\max _{i} \phi_{i}, \quad \phi=\sum_{i} \phi_{i} .
$$

The arrival instants of the packets also remain the same in both systems. Note that these packets can arrive from any flow.

Our sample path analysis is along the lines of [2] and [4] and the notations are similar to that in [4]—we consider identical input sequences to both the GPS and the WFQ systems and analyze the discrepancies. Packets are numbered according to their departure times in the WFQ system.

Let $\hat{d}_{k}$ be the time at which the $k$-th packet, $p_{k}$, departs from the WFQ system, $a_{k}$ its arrival time to both the systems and $d_{k}$ its departure time in the GPS system and $L_{k}$ the length of the packet. Let $\hat{s}_{k}$ be the time at which the transmission of packet $p_{k}$ starts in the WFQ system.

The following lemma will be useful in the proof of the theorem and is a fairly straightforward consequence of the definition of WFQ. See [1], [2], [4] for a proof.

Lemma 1: If packets $p_{k_{1}}$ and $p_{k_{2}}$ are present in the system at a scheduling instant and if packet $p_{k_{1}}$ is scheduled to transmit before packet $p_{k_{2}}$ in WFQ, then packet $p_{k_{1}}$ departs before packet $p_{k_{2}}$ in the GPS. This, of course, implies that packet $p_{k_{1}}$ departs before packet $p_{k_{2}}$ in the WFQ system. 


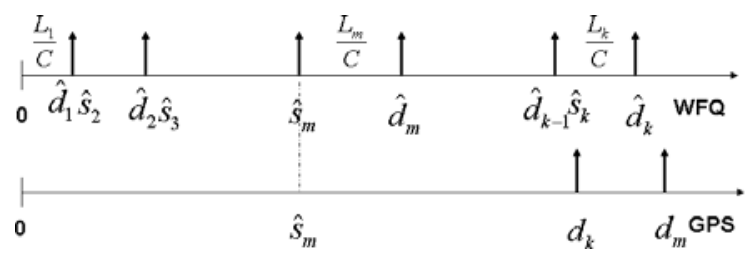

Fig. 1. Events of interest in the WFQ and GPS systems. (Adopted from [4])

The following theorem presents the tighter bound on the departure time of a packet in WFQ and GPS system.

Theorem 1: For all packets in the WFQ scheduler,

$$
\hat{d}_{k} \leq d_{k}+\frac{L_{\max }}{C}-\frac{\left(\frac{\phi_{\min }}{\phi_{\max }}\right) L_{\min }}{C} .
$$

Proof: Since both the GPS and WFQ are work conserving queueing disciplines, their busy periods will be identical and it suffices to consider a busy period that starts, without loss of generality, at time 0 . Further, during a busy period, WFQ and GPS schedulers will transmit the same set of packets. Since the packets are indexed according to their departure in the WFQ, $\hat{d}_{1}<\hat{d}_{2}<\cdots$.

Since the busy period starts at 0 , clearly, $\hat{d}_{k}=\frac{\sum_{i=1}^{k} L_{i}}{C}$. We need to consider two cases for the ordering of the departure times of packets in GPS relative to that in WFQ.

Case 1: None of the packets $p_{1}, p_{2}, \ldots, p_{k-1}$ departs after $d_{k}$ in GPS. Hence, in GPS, at least $\sum_{i=1}^{k} L_{i}$ bits have been transmitted up to $d_{k}$ and we have

$$
d_{k} \geq \frac{\sum_{i=1}^{k} L_{i}}{C}
$$

Since $\hat{d}_{k}=\frac{\sum_{i=1}^{k} L_{i}}{C}$, the theorem is trivially true.

Case 2: There are some packets that depart earlier in WFQ than in GPS. These packets consume bandwidth that was meant for other packets and hence delay them. Consider such a packet $p_{m}, 1 \leq m \leq k-1$ that has the largest index, such that $d_{m}>d_{k}$. Of the packets that should have departed after packet $p_{k}, p_{m}$ is the last packet that departs earlier than $p_{k}$ in WFQ and packets $p_{m+1}, \ldots, p_{k-1}$ depart earlier than packet $p_{k}$ in both WFQ and GPS. This means, $d_{m+1} \leq d_{k}, d_{m+2} \leq d_{k}, \ldots, d_{k-1} \leq d_{k}$. By Lemma 1 , packets $p_{m+1}, p_{m+2}, \ldots, p_{k}$ were not present in the WFQ system at time $\hat{s}_{m}$, because if they were, one of them would have been chosen for service instead of $p_{m}$. Figure 1 shows an example situation.

In the interval $\left[\hat{s}_{m}, d_{k}\right]$, GPS has transmitted complete packets $p_{m+1}, \ldots, p_{k}$. In the same interval, it would have also served some bits of packet $p_{m}$. Let $L_{m}^{\prime}$ be the minimum number of bits of packet $p_{m}$ that was transmitted by GPS in this interval $\left[\hat{s}_{m}, d_{k}\right]$. Hence,

$$
d_{k} \geq \hat{s}_{m}+\frac{\sum_{i=m+1}^{i=k} L_{i}}{C}+\frac{L_{m}^{\prime}}{C} .
$$

Let $f_{k}$ and $f_{m}$ be the flow to which packet $p_{k}$ and $p_{m}$ belong respectively. Since packet $p_{k}$ was completely transmitted by GPS in the interval $\left[\hat{s}_{m}, d_{k}\right]$, (it arrived after $\hat{s}_{m}$ and departed at $d_{k}$ ) the number of bits of packet $p_{m}$ that were transmitted in this interval is at least $\frac{\phi_{f_{m}}}{\phi_{f_{k}}} L_{k}$, i.e.,

$$
L_{m}^{\prime} \geq \frac{\phi_{f_{m}}}{\phi_{f_{k}}} L_{k}
$$

From Figure 1, it is clear that

$$
\hat{d}_{k}=\hat{s}_{m}+\frac{L_{m}}{C}+\frac{\sum_{i=m+1}^{k} L_{i}}{C} .
$$

Using (3) and (4) in (2), we get

$$
d_{k} \geq \hat{d}_{k}-\frac{L_{m}}{C}+\frac{\phi_{f_{m}}}{\phi_{f_{k}}} \frac{L_{k}}{C} .
$$

But $L_{m} \leq L_{\max }$ and $\left(\frac{\phi_{f_{m}}}{\phi_{f_{k}}}\right) L_{k} \geq\left(\frac{\phi_{\min }}{\phi_{\max }}\right) L_{\min }$. Hence, from (5), we get

$$
d_{k} \geq \hat{d}_{k}-\frac{L_{\max }}{C}+\frac{\left(\frac{\phi_{\min }}{\phi_{\max }}\right) L_{\min }}{C}
$$

and the theorem follows.

This delay bound also impacts the fairness discrepancy of WFQ. Let $U^{j}(t)$ and $\hat{U}^{j}(t)$ be the number of bits of flow $j$ transmitted by GPS and WFQ respectively up to time $t$. [1], [2] have shown that for all $t$ and for all $j, U^{j}(t)-\hat{U}^{j}(t) \leq L_{\max }$. Theorem 2 below shows that this discrepancy is tighter if the link prescribes a minimum packet length.

Theorem 2:(i) Let $L_{k}^{j}$ denote the number of bits in $k$-th packet of flow $j, p_{k}^{j}$. The fairness discrepancy between the WFQ and GPS systems satisfies the following inequality. For $\hat{d}_{k-1}^{j} \leq t \leq \hat{d}_{k}^{j}$

$$
U^{j}(t)-\hat{U}^{j}(t) \leq\left\{\begin{array}{c}
L_{\max }-\frac{\phi_{\min }}{\phi_{\max }} L_{\min } \\
\quad \text { if } L_{k}^{j} \leq L_{\max }-\frac{\phi_{\min }}{\phi_{\max }} L_{\min } \\
L_{k}^{j}-\left(\frac{\phi_{j}}{\phi}\left|L_{\max }-L_{k}^{j}-\frac{\phi_{\min }}{\phi_{\max }} L_{\min }\right|\right) \\
\quad \text { if } L_{k}^{j} \geq L_{\max }-\frac{\phi_{\min }}{\phi_{\max }} L_{\min }
\end{array}\right.
$$

(ii) Further, for all $t$

$$
U^{j}(t)-\hat{U}^{j}(t) \leq L_{\max }-\frac{\phi_{\min }}{\phi_{\max }} \frac{\phi_{j}}{\phi} L_{\min }
$$

for all $t \geq 0$ and for $j=1, \ldots, N$.

Proof: Consider packet $p_{k}^{j}$. Since packets within a flow are served in FIFO order, the total number of bits of flow $j$ until the departure of packet $p_{k}^{j}$ under the two systems should be equal, i.e.,

$$
U^{j}\left(d_{k}^{j}\right)=\hat{U}^{j}\left(\hat{d}_{k}^{j}\right)
$$

Since WFQ is a packet based scheduler, the entire link bandwidth is dedicated to a packet. Hence,

$$
\hat{U}^{j}\left(\hat{d}_{k}^{j}\right)=\hat{U}^{j}\left(\hat{s}_{k}^{j}\right)+L_{k}^{j},
$$

From Theorem 1 we have

$$
d_{k}^{j} \geq \hat{d}_{k}^{j}-\left(\frac{L_{\max }}{C}-\frac{\frac{\phi_{\min }}{\phi_{\max }} L_{\min }}{C}\right) .
$$

Changing signs, and hence the direction of the above inequality, and adding $\hat{s}_{k}^{j}$ to both sides, we get

$$
\hat{s}_{k}^{j}-d_{k}^{j} \leq \hat{s}_{k}^{j}-\hat{d}_{k}^{j}+\frac{L_{\max }}{C}-\frac{\frac{\phi_{\min }}{\phi_{\max }} L_{\min }}{C} .
$$


Since $\hat{d}_{k}^{j}-\hat{s}_{k}^{j}=\frac{L_{k}^{j}}{C}$, the above inequality can be rewritten as

$$
\hat{s}_{k}^{j} \leq d_{k}^{j}+\frac{L_{\max }-L_{k}^{j}-\frac{\phi_{\min }}{\phi_{\max }} L_{\min }}{C} .
$$

Denote $Z_{k}^{j}:=L_{\max }-L_{k}^{j}-\frac{\phi_{\min }}{\phi_{\max }} L_{\min }$. Since both $U^{j}(t)$ and $\hat{U}^{j}(t)$ (and also the departure time of packets in both the systems) are non-decreasing, we can write the following inequality.

$$
U^{j}\left(\hat{s}_{k}^{j}\right) \leq U^{j}\left(d_{k}^{j}+\frac{Z_{k}^{j}}{C}\right)
$$

Using (10) in (12) we get

$$
U^{j}\left(\hat{s}_{k}^{j}\right)-\hat{U}^{j}\left(\hat{s}_{k}^{j}\right)-L_{k}^{j} \leq U^{j}\left(d_{k}^{j}+\frac{Z_{k}^{j}}{C}\right)-\hat{U}^{j}\left(\hat{d}_{k}^{j}\right) .
$$

$Z_{k}^{j}$ could be positive or negative and we consider these cases separately.

Case 1: $Z_{k}^{j} \geq 0$. In this case $U^{j}\left(d_{k}^{j}+\frac{Z_{k}^{j}}{C}\right)$ will be upper bounded by $U^{j}\left(d_{k}^{j}\right)+Z_{k}^{j}$. This is because the maximum number of bits that can be transmitted in time $Z_{k}^{j} / C$ is $Z_{k}^{j}$. Using this bound and (9) in (13), we get

$$
U^{j}\left(\hat{s}_{k}^{j}\right)-\hat{U}^{j}\left(\hat{s}_{k}^{j}\right) \leq L_{k}^{j}+Z_{k}^{j} .
$$

Substituting for $Z_{k}^{j}$ we get

$$
U^{j}\left(\hat{s}_{k}^{j}\right)-\hat{U}^{j}\left(\hat{s}_{k}^{j}\right) \leq L_{\max }-\frac{\phi_{\min }}{\phi_{\max }} L_{\min } .
$$

Case 2: $Z_{k}^{j}<0$. In this case a simple upper bound on $U^{j}\left(d_{k}^{j}+\frac{Z_{k}^{j}}{C}\right)$ would be $U^{j}\left(d_{k}^{j}\right)$ and

$$
U^{j}\left(\hat{s}_{k}^{j}\right)-\hat{U}^{j}\left(\hat{s}_{k}^{j}\right) \leq L_{k}^{j}
$$

This can be further tightened by upper bounding $U^{j}\left(d_{k}^{j}+\frac{Z_{k}^{j}}{C}\right)$ by $U^{j}\left(d_{k}^{j}\right)-W_{\min }\left(\frac{Z_{k}^{j}}{C}\right)$ where $W_{\min }\left(\frac{Z_{k}^{j}}{C}\right)$ is the minimum work that will be done in the interval $\frac{\left|Z_{k}^{j}\right|}{C}$ in the GPS system. From (13), we get

$$
\begin{aligned}
U^{j}\left(\hat{s}_{k}^{j}\right)-\hat{U}^{j}\left(\hat{s}_{k}^{j}\right) & \leq L_{k}^{j}+U^{j}\left(d_{k}^{j}\right)-\hat{U}^{j}\left(\hat{d}_{k}^{j}\right)-W_{\min }\left(\frac{Z_{k}^{j}}{C}\right) \\
& \leq L_{k}^{j}-\left(\frac{\phi_{j}}{\phi}\left|Z_{k}^{j}\right|\right)
\end{aligned}
$$

In obtaining the last equality above, we have used (9) and the fact that minimum number bits of flow $j$ that is transmitted in the GPS system in a time interval $T$ is $\left(\frac{\phi_{j}}{\phi}\right) T C$.

The slope of $\hat{U}^{j}$ alternates between $C$ when a packet from flow $j$ is served and 0 when flow $j$ is not being served. Since the slope of $U^{j}$ also follows the same limits, for $\hat{d}_{k-1} \leq t<$ $\hat{d}_{k}$, the difference $U^{j}(t)-\hat{U}^{j}(t)$ has its maximum value at $\hat{s}_{k}$, when the packet of flow $j$ starts service in WFQ. Thus part (i) of the theorem holds.

To prove part (ii) consider the case of $Z_{k}^{j}<0$, when the fairness discrepancy is given by (15). Note that $Z_{k}^{j}<0$ occurs while $Z_{k}^{j} \geq 0$ need not occur. Now observe that $\left|Z_{k}^{j}\right|$ increases linearly with $L_{k}^{j}$ when $Z_{k}^{j}<0$ and that the slope of $\left(\frac{\phi_{j}}{\phi}\left|Z_{k}^{j}\right|\right)$ is $\frac{\phi_{j}}{\phi} \leq 1$. In the RHS of (15), we are subtracting $\left(\frac{\phi_{j}}{\phi}\left|Z_{k}^{j}\right|\right)$ from $L_{k}^{j}$. Hence the maximum value of the RHS in (15) occurs when $L_{k}^{j}=L_{\max }$ and we have

$$
U^{j}\left(\hat{s}_{k}^{j}\right)-\hat{U}^{j}\left(\hat{s}_{k}^{j}\right) \leq L_{\max }-\frac{\phi_{\min }}{\phi_{\max }} \frac{\phi_{j}}{\phi} L_{\min }
$$

Since $\frac{\phi_{j}}{\phi} \leq 1$, the bound obtained above for $Z_{k}^{j}<0$ is looser than that obtained for $Z_{k}^{j} \geq 0$ in (14) and part (ii) of the theorem follows.

\section{DISCUSSION}

It is instructive to consider some special cases to see the impact of the tighter bounds on the discrepancy. First consider the case when all the flows have equal weight, i.e., $\phi_{\max }=$ $\phi_{\min }$. Then (1) says that the delay discrepancy between the WFQ and GPS schedulers is upper bounded by $\frac{L_{\max }-L_{\min }}{C}$.

A second case of interest is that of fixed size packets, i.e., $L_{\max }=L_{\min }$ for which the delay discrepancy is upper bounded by $\left(1-\frac{\phi_{\min }}{\phi_{\max }}\right) L_{\max }$. This can be substantially smaller than the $L_{\max }$ bound. This case is of special interest because most streaming traffic (e.g., VoIP) use fixed size packets. Note that, if in this system $\phi_{\min }=\phi_{\max }$ then the delays in the WFQ system is never greater than the delay in the GPS system.

\section{REFERENCES}

[1] A. Greenberg and N. Madras, "How fair is fair queuing?," Journal of the ACM, vol. 39, pp. 568-598, July 1992.

[2] A. Parekh and R. G. Gallager, "A generalised processor sharing approach to flow control in integrated services networks: the single-node case," IEEE/ACM Trans. Networking, vol. 1, pp. 344-357, June 1993.

[3] A. Demers, S. Keshav, and S. Shenker, "Analysis and simulation of a fair queueing algorithm," Internetworking Res. and Exp., vol. 1, pp. 3 26, 1990.

[4] A. Kumar, D. Manjunath, and J. Kuri, Communication Networking - An Analytical Approach. Morgan Kaufmann, 2004.

[5] S. J. Golestani, "A self clocked fair queueing scheme for broadband applications," in Proc. IEEE INFOCOM 1994, pp. 636-646.

[6] J. C. Bennett and H. Zhang, "Hierarchical fair queuing algorithms," IEEE/ACM Trans. Networking, vol. 5, pp. 675-689, Oct. 1997.

[7] P. Goyal, H. M. Vin, and H. Cheng, "Start-time fair queueing: a scheduling algorithm for integrated services packet switching networks," IEEE/ACM Trans. Networking, vol. 5, pp. 690-704, Oct. 1997.

[8] A. Varma and D. Stiliadis, "Hardware implementation of fair queueing algorithms for asynchronous transfer networks," IEEE Commun. Mag., vol. 35, no. 12, pp. 54-68, Dec. 1997.

[9] D. Stiliadis and A. Varma, "Efficient fair queueing algorithms for packetswitched networks," IEEE/ACM Trans. Networking, vol. 6, pp. 175-185, April 1998. 